\title{
Intercellular junctions in Ewing sarcoma/ primitive neuroectodermal tumor: additional evidence of epithelial differentiation
}

\author{
Audrey N Schuetz ${ }^{1}$, Brian P Rubin ${ }^{2}$, John R Goldblum ${ }^{3}$, Bahig Shehata ${ }^{1}$, Sharon W Weiss ${ }^{1}$, \\ Wendy Liu ${ }^{3}$, Mark R Wick ${ }^{4}$ and Andrew L Folpe ${ }^{1}$ \\ ${ }^{1}$ Department of Pathology and Laboratory Medicine, Emory University, Atlanta, GA, USA; ${ }^{2}$ Department of \\ Pathology, University of Washington Medical Center, Seattle, WA, USA; ${ }^{3}$ Department of Pathology, Cleveland \\ Clinic Foundation, Cleveland, OH, USA and ${ }^{4}$ Department of Pathology, University of Virginia, \\ Charlottesville, VA, USA
}

\begin{abstract}
Ewing sarcoma/primitive neuroectodermal tumor (ES/PNET) has recently been shown to frequently express cytokeratins, suggesting partial epithelial differentiation. Older ultrastructural studies have documented primitive cell-cell junctions in ES/PNET, reportedly resembling poorly formed desmosomes. Recently, paraffinreactive antibodies have become available to proteins found in a variety of intercellular junctions indicative of epithelial differentiation, including tight junctions, desmosomes and adherens junctions. We examined intercellular junction protein expression in a large number of genetically confirmed ES/PNET. Formalin-fixed, paraffin-embedded sections from $\mathbf{2 3}$ primary and seven recurrent or metastatic cases of genetically confirmed ES/PNET were immunostained for claudin-1 and occludin (tight junction structural proteins), zonula occludens1 (ZO-1, tight junction linker protein), desmoglein 1/2 (desmosomal adherens protein), desmoplakin (desmosomal structural protein) and E-cadherin (epithelial adherens junction protein), using steam heatinduced epitope retrieval and the Dako Envision system. Cases with $>5 \%$ positive cells were scored as 'positive'. Normal colonic epithelium and skin served as external positive controls. Claudin-1 was expressed by 19 of 30 specimens (63\%), ZO-1 was expressed by 15 of 29 specimens $(51 \%)$, and occludin was expressed by three of 28 specimens (11\%). In 28 specimens all three tight junction markers were evaluable. In all, 15 samples (54\%) expressed only one tight junction marker, and 10 samples $(36 \%)$ expressed two tight junction markers. No case expressed all three tight junction markers. Desmoglein was expressed in one of $30(3 \%)$ samples. Desmoplakin was expressed in two of $28(7 \%)$ samples. E-cadherin was negative in all cases. Our data suggest that many of the previously described cell-cell junctions in ES/PNET are poorly formed tight junctions, given the high frequency of claudin-1 and ZO-1 expression. This may underestimate the true frequency of tight junction protein expression in ES/PNET, as there are at least $\mathbf{2 0}$ different claudins and other ZO proteins. These tight junctions are almost certainly abnormal, given the absence of occludin expression in most cases. Desmosomal and adherens junction protein expression was rare to absent. Our findings provide additional evidence that ES/PNET frequently show partial epithelial differentiation.
\end{abstract}

Modern Pathology (2005) 18, 1403-1410. doi:10.1038/modpathol.3800435; published online 6 May 2005

Keywords: Ewing sarcoma; primitive neuroectodermal tumor; tight junction; desmosome; adherens junction

Ewing sarcoma/primitive neuroectodermal tumor (ES/PNET) is a primitive bone and soft tissue sarcoma that may occur in both children and adults. Although ES/PNET has traditionally been considered to be of neuroectodermal derivation, it gener-

Correspondence: Dr AL Folpe, MD, Department of Pathology, H-175, Emory University Hospital, 1364 Clifton Road NE, Atlanta, GA 30322, USA.

E-mail: afolpe@emory.edu

Received 24 February 2005; revised 11 March 2005; accepted

1 April 2005; published online 6 May 2005 ally does not show evidence of any specific line of differentiation, either by ultrastructural or immunohistochemical methods. ${ }^{1-9}$

Over the last two decades it has been discovered that over $85 \%$ of ES/PNET have a specific and diagnostic translocation, $\mathrm{t}(11 ; 22)(\mathrm{q} 24 ; \mathrm{q} 12)$ that results in the formation of an EWS/FLI-1 fusion protein, with a minority of ES/PNET having other specific translocations, including $\mathrm{t}(21 ; 22), \mathrm{t}(17 ; 22)$, and $t(2 ; 22) \cdot{ }^{9-11}$ These ES/PNET-specific genetic events can be identified either through traditional cytogenetic methods or with the assistance of 
molecular genetic techniques, such as RT-PCR and FISH, and are undoubtedly critical to its pathogenesis.

Recently, it has been shown that up to $25 \%$ of ES/ PNET express cytokeratins, suggestive of partial epithelial differentiation. ${ }^{12,13}$ These findings are intriguing, inasmuch as older ultrastructural studies of ES/PNET have documented primitive intercellular junctions, ${ }^{5,7}$ and a recent study has documented particularly frequent intercellular junction formation in cytokeratin-positive ES/PNET. ${ }^{14}$ A single immunohistochemical study has found expression of desmosomal proteins, such as desmoplakin and plakoglobin ( $\alpha$-catenin) in these primitive junctions and suggested that this finding was indicative of at least some epithelial differentiation in ES/PNET. ${ }^{15}$

Paraffin-reactive antibodies have lately become available to proteins found in a variety of intercellular junctions, including tight junctions, desmosomes, and adherens junctions. We studied a large number of genetically confirmed cases of ES/PNET with markers to the tight junction structural proteins claudin-1 and occludin, the tight junction linker protein zonula occludens-1 (ZO-1), the desmosomal cadherins desmoglein $1 / 2$, the desmosomal structural protein desmoplakin, and the epithelial cell adherens junction protein E-cadherin, in order to more fully characterize their intercellular junctions.

\section{Materials and methods}

Formalin-fixed, paraffin-embedded blocks from 31 specimens (24 primaries, seven recurrences/metastases from these 24 primaries) of ES/PNET were retrieved from the archives of Emory University, the University of Washington Medical Center, and the Cleveland Clinic Foundation. All cases had previously been shown to be positive for the ES/PNETspecific translocations $\mathrm{t}(11 ; 22)$ (23 cases) or $\mathrm{t}(21 ; 22)$ (one case) by traditional cytogenetic study, RT-PCR or FISH (data not shown). Outcome data were not available.

All cases were immunostained at Emory University with the following commercially available antibodies: claudin-1 (polyclonal, 1:70, Zymed Laboratories); ZO-1 (Clone 1, 1:400, Transduction Laboratories); occludin (Clone OC-3F10, 1:100, Santa Cruz Biotechnology); desmoglein 1/2 (DG 3.10, 1:15, Research Diagnostics); and E-cadherin (ECCD-2, 1:200, Zymed Laboratories). Steam heatinduced epitope retrieval and the Dako Envision system were used. Normal colonic epithelium, skin and endothelial cells served as positive controls. Negative controls consisted of substitution of buffer for the primary antibody. Cases were scored as 'positive' if greater than $5 \%$ of cells stained positively. Only membranous staining was scored. This $5 \%$ cutoff was arrived at after initial review of

Table 1 Immunohistochemical results

\begin{tabular}{|c|c|c|c|c|c|c|}
\hline Specimen number & Claudin-1 & $Z O-1$ & Occludin & Desmoplakin & Desmoglein 1/2 & E-Cadherin \\
\hline 1 & Negative & Negative & Negative & Negative & Negative & Negative \\
\hline 2 & Negative & Negative & Negative & Negative & Negative & Negative \\
\hline 3 & Negative & Positive & No tumor & Negative & Negative & Negative \\
\hline 4 & No tumor & No tumor & No tumor & Negative & Negative & Negative \\
\hline 5 & Positive & No tumor & No tumor & Positive & No tumor & Negative \\
\hline 6 & Positive & Negative & Negative & Negative & Negative & Negative \\
\hline 7 & Positive & Negative & Negative & Negative & Negative & Negative \\
\hline 8 & Positive & Negative & Negative & Negative & Negative & Negative \\
\hline 9 & Positive & Negative & Negative & Negative & Negative & Negative \\
\hline 10 & Positive & Negative & Negative & Negative & Negative & Negative \\
\hline 11 & Positive & Negative & Negative & Negative & Negative & Negative \\
\hline 12 & Positive & Positive & Negative & Negative & Negative & Negative \\
\hline 13 & Positive & Positive & Negative & Positive & Negative & Negative \\
\hline 14 & Positive & Positive & Negative & Negative & Negative & Negative \\
\hline 15 & Positive & Negative & Negative & Negative & Negative & Negative \\
\hline 16 & Positive & Negative & Negative & Negative & Negative & Negative \\
\hline 17 & Negative & Negative & Negative & Negative & Negative & Negative \\
\hline $18 \mathrm{a}$ & Negative & Positive & Positive & Negative & Negative & Negative \\
\hline $18 \mathrm{~b}$ & Negative & Positive & Positive & Negative & Negative & Negative \\
\hline $18 \mathrm{c}$ & Negative & Positive & Negative & Negative & Negative & Negative \\
\hline $18 \mathrm{~d}$ & Positive & Positive & Negative & Positive & Positive & Negative \\
\hline 19 & Positive & Positive & Negative & Negative & Negative & Negative \\
\hline $20 a$ & Negative & Positive & Negative & Negative & Negative & Negative \\
\hline $20 \mathrm{~b}$ & Negative & Positive & Negative & Negative & Negative & Negative \\
\hline $21 a$ & Positive & Positive & Negative & Negative & Negative & Negative \\
\hline $21 b$ & Positive & Negative & Positive & Positive & Negative & Negative \\
\hline $22 a$ & Positive & Positive & Negative & Negative & Negative & Negative \\
\hline $22 b$ & Positive & Negative & Negative & Negative & Negative & Negative \\
\hline 23 & Negative & Positive & Negative & Negative & Negative & Negative \\
\hline 24 & Positive & Positive & Negative & Positive & Negative & Negative \\
\hline
\end{tabular}


the immunostained slides, which showed these cell junction markers to frequently be expressed in a patchy fashion in clusters of cells, rather than diffusely. All case were independently scored by two observers (ANS and ALF) and any differences were resolved by consensus.

Immunostaining for desmoplakin was performed at the University of Virginia (DP 2.17, 1:10, Research Diagnostics Inc.) using microwave oven-induced epitope retrieval and the Dako Envision system. Normal epidermal keratinocytes served as a positive external control.

\section{Results}

The immunohistochemical results are detailed in Table 1. In all cases, positive staining for the various proteins was membranous and circumferential (nonpolarized). In general, positive cases showed expression of ZO-1 and claudin-1 in $>50 \%$ of cells. In contrast, expression of occludin, desmoglein and desmoplakin was almost always confined to scattered clusters of positively staining cells.
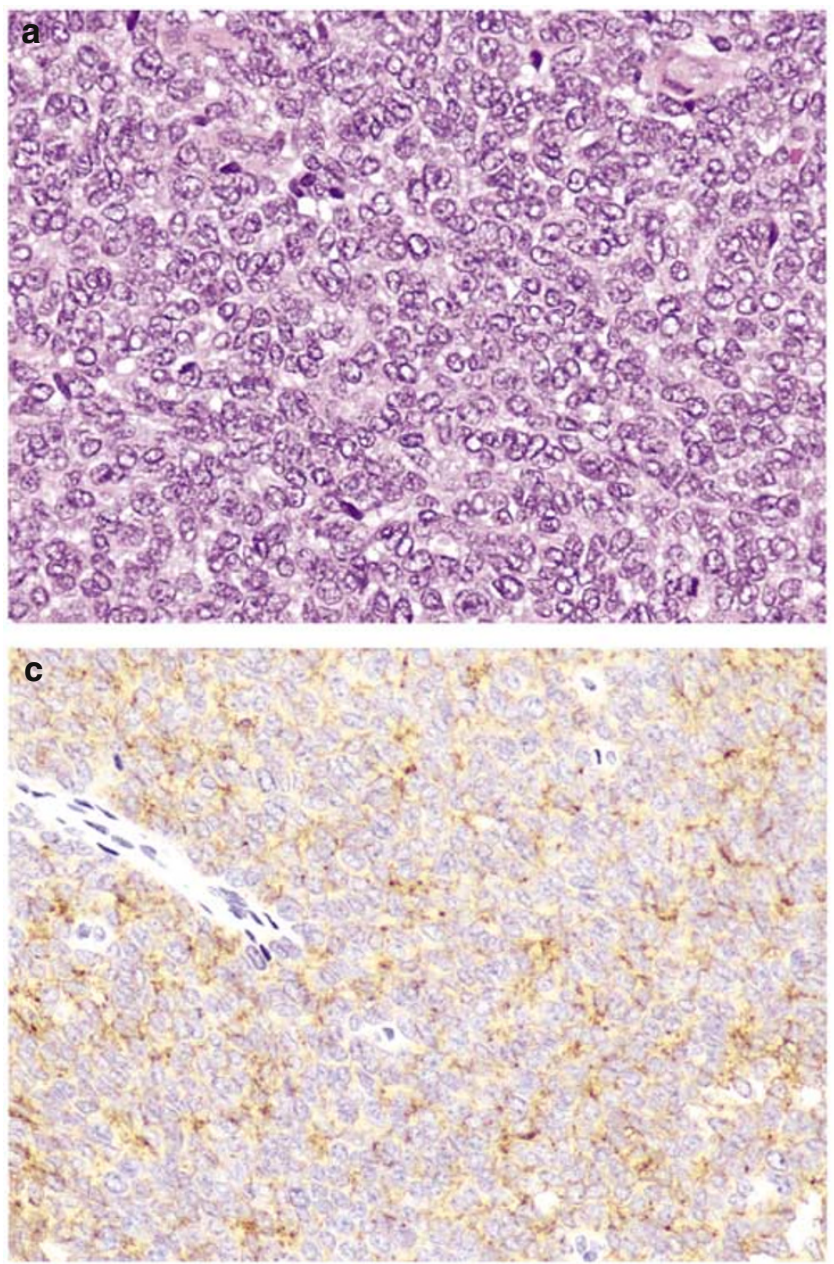

Of the tight junction markers, claudin-1 was expressed by 19 of 30 specimens (63\%), ZO-1 was expressed by 15 of 29 specimens (51\%), and occludin was expressed by three of 28 specimens (11\%). In 28 specimens all three tight junction markers were evaluable. In all, 15 samples (54\%) expressed only one tight junction marker, and 10 samples $(36 \%)$ expressed two tight junction markers. No case expressed all three tight junction markers (Figures 1-3). Desmoglein 1/2 was expressed in one of $30(3 \%)$ cases (Figure 2). Desmoplakin was expressed by two of $28(7 \%)$ of cases; one of these cases (an 'adamantinoma-like' ES/PNET) also expressed ZO-1, claudin-1 and desmoglein $1 / 2$, whereas the other desmoplakinpositive case (a conventional ES/PNET) expressed ZO-1 and claudin-1, but not desmoglein $1 / 2$ or occludin (Figure 4). E-cadherin was negative in all 30 samples tested.

\section{Discussion}

Intercellular junctions allow individual cells to remain structurally linked to each other, while
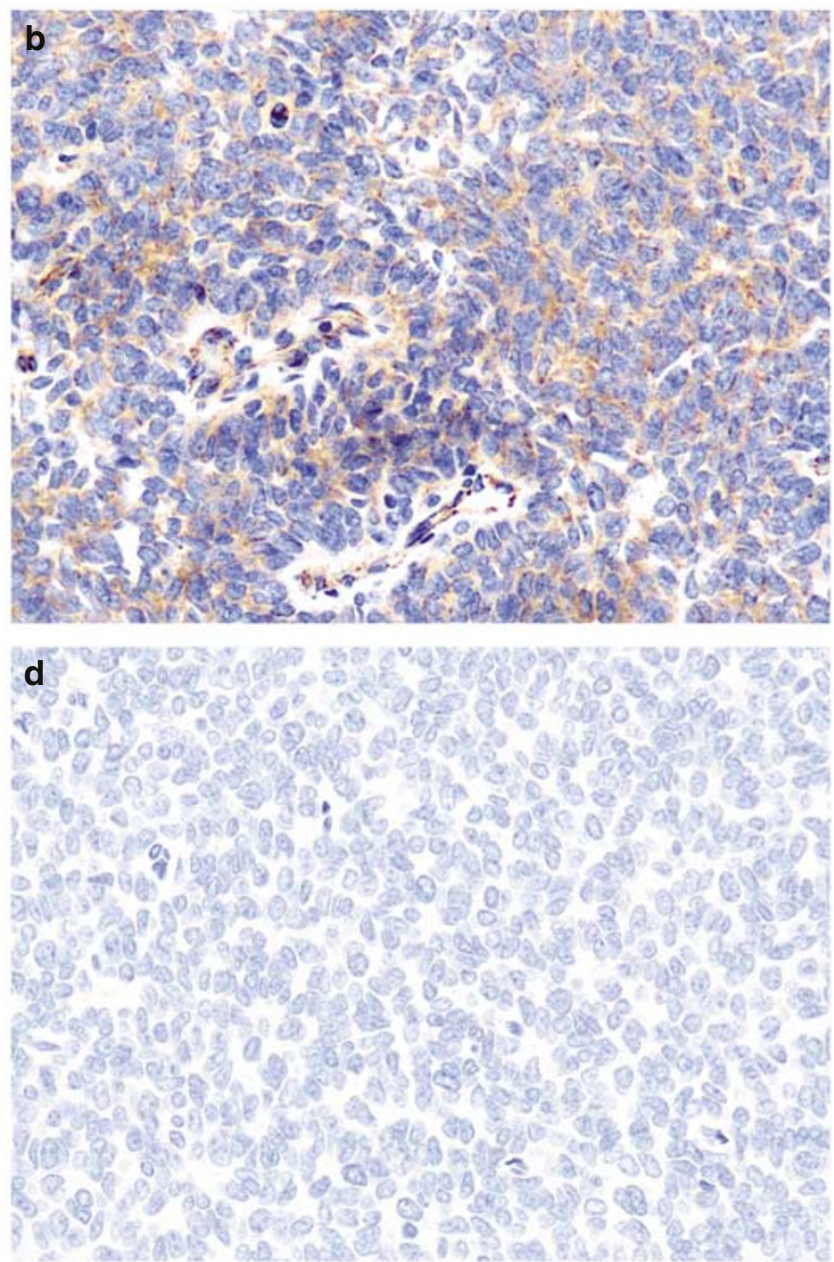

Figure 1 Typical ES/PNET (a) showing strong expression of ZO-1 (b) and claudin-1 (c), but not occludin (d). The absence of occludin expression is suggestive of abnormal tight junction formation. 

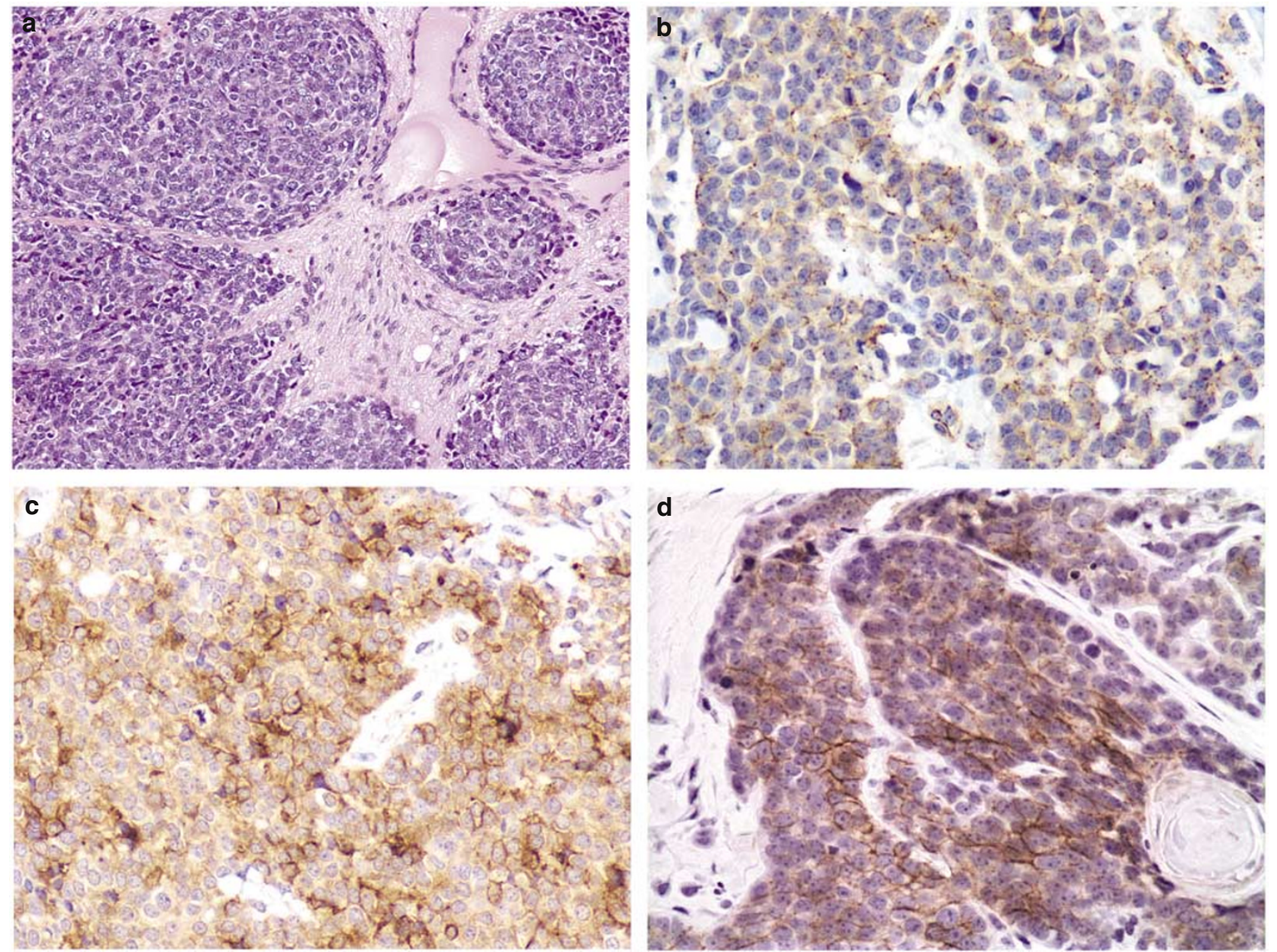

Figure 2 'Adamantinoma-like' ES/PNET showing pronounced nested growth and peripheral nuclear palisading (a). This case showed strong expression of ZO-1 (b), claudin-1 (c) and desmoglein 1/2 (d). This case also showed focal expression of desmoplakin (not shown).

controlling the diffusion of solutes between adjacent cells. Such mechanical linkages among cells create boundaries and compartments, which are fundamental in multicellular organisms. Several types of intercellular junctions exist including tight junctions (most apically located), adherens junctions and desmosomes (most basally located).

Although Friedman and Gold ${ }^{2}$ did not identify intercellular junctions in their seminal 1968 ultrastructural study of ES/PNET, such junctions were noted shortly thereafter by a number of investigators, including Hou-Jensen et $a l^{3}{ }^{3}$ Takayama and Sugawa $^{16}$ and Llombart-Bosch et al. ${ }^{5}$ In general, these junctions have been described as rudimentary, resembling desmosomes or 'desmosome-like junctions', although tight junction-like structures have also rarely been reported. ${ }^{5}$ Perhaps the most detailed ultrastructural study of intercellular junctions in ES/PNET is that of Navas-Palacios et al, ${ }^{7}$ who noted intercellular junctions in $70 \%$ of 33 studied ES/ PNET, with well-developed desmosomes and tight junctions noted in only $9 \%$ of cases, and primitive junctions of indeterminate type present in over 90\% of cases. Most recently, Srivastava et $a 1^{14}$ have documented cell-cell junctions of uncertain type in 12 cases of cytokeratin-positive ES/PNET.

The overwhelming majority of studies of ES/ PNET over the last 20 years have concentrated on its immunophenotype and the molecular genetic events underlying its pathogenesis, and relatively little has been added to our understanding of these enigmatic intercellular junctions. The only study we are aware of that has attempted to further characterize the intercellular junctions of ES/PNET is that of Moll et $a,^{15}$ who demonstrated at least focal expression of desmoplakin (a desmosomal protein), vinculin (a desmosomal and adherens junction protein) and plakoglobin (a desmosomal protein) in all 11 ES/PNET studied. The desmosomal cadherin, desmoglein, was negative in the three studied cases, and tight junction-related and adherens junction-related proteins were not studied.

In epithelial cells, tight junctions define membrane polarity, form an intercellular seal, and 

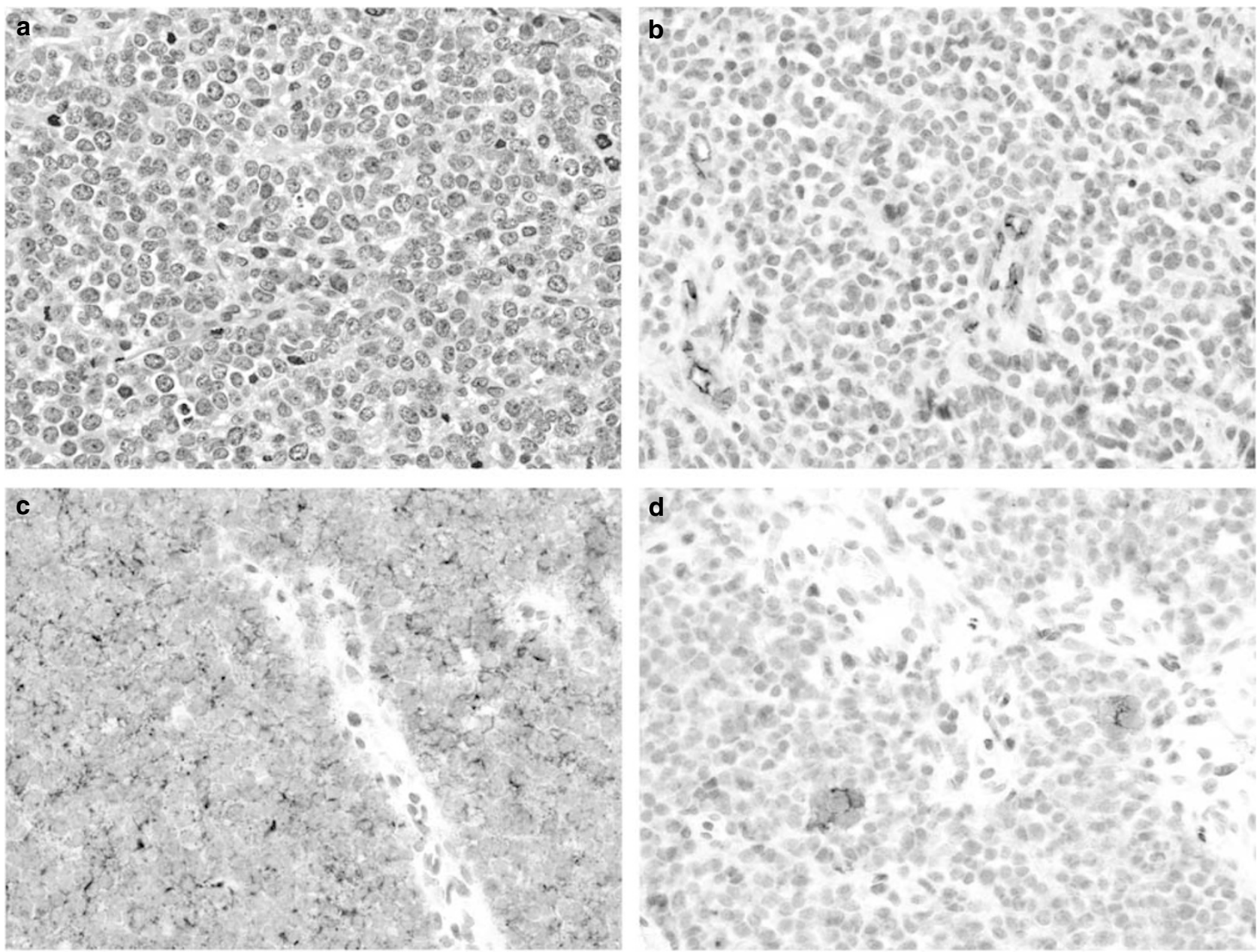

Figure 3 Typical ES/PNET (a), negative for ZO-1 (b), but positive for claudin-1 (c) and occludin (d).

regulate paracellular transport of water, solutes and cells. ${ }^{17}$ The molecular constituents of tight junctions include integral transmembrane proteins, such as the claudin family, occludin, and junctional adhesion molecule (JAM), and scaffolding or linker proteins, such as the zonula occludens family. ${ }^{18}$ Tight junctions also appear to play a critical role in signal transduction through interactions with the actin cytoskeleton. ${ }^{17}$ Tight junctions are largely restricted to epithelial cells, although they are also present in endothelium and perineurial cells. ${ }^{19}$

The claudins are a family of approximately 20 homologous proteins that help determine tight junction structure and permeability, and which appear to be differentially expressed in tissues, with claudin-1 expression, for example, being relatively widespread among epithelia, and claudin-3 expression being confined to lung and liver epithelia. ${ }^{18}$ Occludin, a structural and functional component of the tight junction, has been identified in most epithelial tight junctions, at both the gene and protein level, although its role in assembly and maintenance of tight junctions is still undefined. ${ }^{20-22}$
As far as is known, all normal tight junctions contain occludin, although there is some experimental evidence to suggest that occludin may not be obligatory for tight junction formation. ${ }^{20} \mathrm{ZO}-1$ (zonula occludens-1) is a scaffolding protein that links the transmembrane tight junction proteins to cytoplasmic proteins and the actin cytoskeleton. ${ }^{23,24}$ Unlike the claudins and occludin, $\mathrm{ZO}-1$ is not confined to the tight junction and also plays a role in the formation of the adherens junction. ${ }^{25,26}$. Thus, ZO-1 is expressed in a variety of nonepithelial cell types including Schwann cells, dermal fibroblasts, and astrocytes. ${ }^{27-29}$

Although Navas-Palacios noted tight junctions in only $9 \%$ of ES/PNET by electron microscopy, our data strongly suggests that these junctions are far more common. Specifically, we noted expression of the tight junction-related proteins claudin-1 and ZO-1 in over $50 \%$ of samples respectively, with coexpression in nearly $40 \%$, and expression of at least one protein in close to $90 \%$ of samples. Although it might be argued that ZO-1 expression is not necessarily indicative of tight junction formation, given its other roles in cell signaling, 
1408
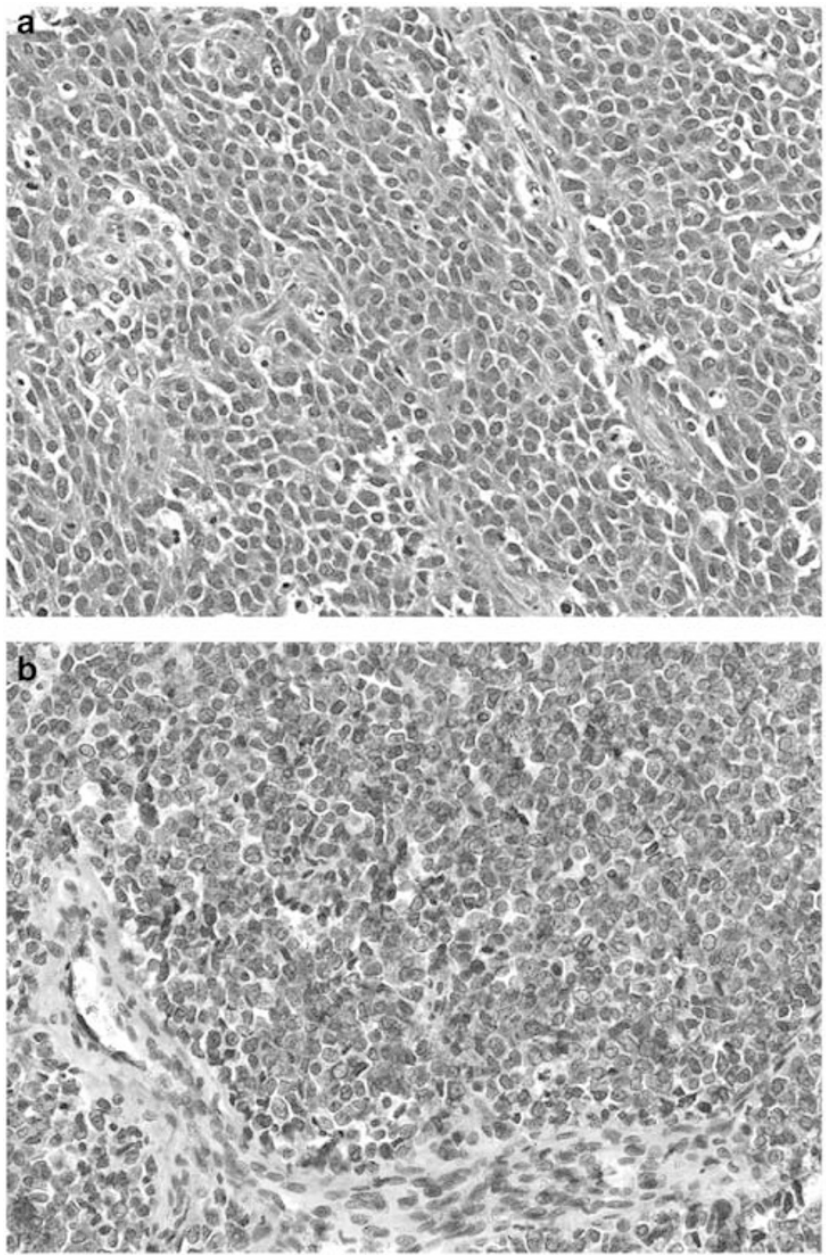

Figure 4 Typical ES/PNET (a) with strong expression of desmoplakin (b). This was the only case to show this degree of desmoplakin expression.

claudin expression is thought to be tight junctionspecific. In fact, our study may underestimate the true frequency of tight junction protein expression in ES/PNET, as there are at least 20 different claudins and $3 \mathrm{ZO}$ proteins. Interestingly, occludin was present in only $11 \%$ of samples, implying that these tight junctions are abnormal, inasmuch as occludin is thought to be present in all known normal tight junctions. These tight junctions have a circumferential, nonpolarized, pattern of distribution, providing further evidence that they are abnormal. We have recently shown a similarly abnormal distribution of tight junctions in another primitive sarcoma, synovial sarcoma. ${ }^{30}$

Desmosomes, along with adherens junctions, comprise the major adhesive junctions of epithelial cells, although they are also present in a variety of nonepithelial tissues, including myocardium, meningeal cells, and ovarian granulosa cells. ${ }^{15,31,32}$ Desmosomes are comprised of three domains; an adhesive transmembrane region, an outer dense protein plaque, and a less dense inner plaque. ${ }^{33}$
The principal components of the adhesive region are the desmosomal cadherins, desmoglein and desmocollin, each of which have three isoforms, with desmoglein and desmocollin present in all desmosomes, and the other isoforms having more restricted patterns of expression. ${ }^{33}$ The outer dense plaque contains plakophilin and plakoglobin, which bind to the major component of the inner plaque, desmoplakin. Desmoplakin is required for the formation of desmosomes and attaches the desmosome to the intermediate filament skeleton. ${ }^{33}$

We have been able to partially confirm the prior observations of Moll et al with regards to desmosomal protein expression. We observed desmoplakin expression in only $7 \%$ of cases, a lower percentage than previously noted. The lower sensitivity of our study is most likely explained by our use of formalin-fixed, paraffin-embedded tissues, as compared with the frozen tissues used by Moll et al. ${ }^{15}$ As with occludin, the absence of desmoglein $1 / 2$ in all but one case also suggests that the desmosomes formed by ES/PNET are abnormal, as all normal desmosomes should contain desmoglein 2 (Dr Otmar Huber, personal communication). The only tumor in this series which expressed both desmoplakin and desmoglein 1/2 was an 'adamantinomalike' ES/PNET, which also expressed high molecular weight cytokeratins (data not shown), suggestive of 'complete' epithelial differentiation. There does not appear to be any correlation between expression of cytokeratins and desmosomal proteins, as a significant subset of the desmosomal proteinnegative cases in the present series were known to express low-molecular weight cytokeratins (data not shown).

The adherens junction plays an important role not only in intercellular adhesion, but also in signal transduction to the actin cytoskeleton. ${ }^{34}$ The transmembrane region of the adherens junction is composed mostly of cadherins, including E-cadherin (epithelia), N-cadherin (neural), R-cadherin (retinal), and P-cadherin (placental). ${ }^{35}$ The cadherins in turn bind to $\alpha$-catenin and $\beta$-catenin, which attach to actin filaments. ${ }^{34,35}$

Although expression of E-cadherin has been documented in a single ES/PNET cell culture, ${ }^{36}$ we are unaware of any other previous study of Ecadherin expression in ES/PNET. We were not able to document E-cadherin expression in any of the 30 samples studied, strongly suggesting that ES/PNET do not form epithelial-type adherens junctions. No previous studies appear to have examined ES/PNET for expression of other cadherins, such as Ncadherin, and this may prove to be an interesting area for future investigation.

In summary, we have found evidence of tight junction protein expression in over $50 \%$ of genetically confirmed ES/PNET, with expression of desmosomal proteins in a small number, and no expression of epithelial adherens junction markers. 
These observations support, we believe, the evolving viewpoint that ES/PNET often show epithelial differentiation, especially when taken in the context of the prior work of Moll and colleagues, the recent observations of cytokeratin expression in a significant number of ES/PNET, and the extremely rare but documented capacity of ES/PNET for complex epithelial differentiation, in the form of so-called 'adamantinoma-like' ES/PNET. Our finding of pan-cytokeratin (AE1/AE3) positivity in 11 of the $24(46 \%)$ cases included in this study (data not shown) and the recent findings of Srivastava et $a l^{14}$ of both cytokeratin expression and cell-cell junction formation in 12 cases of ES/PNET also support this concept. The minor differences in junctional protein expression we observed between primary and recurrent/ metastatic tumors is most likely related to sampling variation, although studies of other primary and metastatic ES/PNET are necessary to more fully address this issue. It remains to be seen whether epithelial differentiation in ES/PNET carries any prognostic significance or provides any information about response to different therapeutic protocols.

\section{References}

1 Ewing J. Diffuse endothelioma of bone. Proc NY Soc Pathol 1921;21:17-24.

2 Friedman B, Gold H. Ultrastructure of Ewing's sarcoma of bone. Cancer 1968;22:307-322.

3 Hou-Jensen K, Priori E, Dmochowski L. Studies on ultrastructure of Ewing's sarcoma of bone. Cancer 1972;29:280-286.

4 Angervall L, Enzinger FM. Extraskeletal neoplasm resembling Ewing's sarcoma. Cancer 1975;36:240-251.

5 Llombart-Bosch A, Blache R, Peydro-Olaya A. Ultrastructural study of 28 cases of Ewing's sarcoma: typical and atypical forms. Cancer 1978;41:1362-1373.

6 Askin FB, Rosai J, Sibley RK, et al. Malignant small cell tumor of the thoracopulmonary region in childhood: a distinctive clinicopathologic entity of uncertain histogenesis. Cancer 1979;43:2438-2451.

7 Navas-Palacios JJ, Aparicio-Duque R, Valdes MD. On the histogenesis of Ewing's sarcoma. An ultrastructural, immunohistochemical, and cytochemical study. Cancer 1984;53:1882-1901.

8 Siebenrock KA, Nascimento AG, Rock MG. Comparison of soft tissue Ewing's sarcoma and peripheral neuroectodermal tumor. Clin Orthop Relat Res 1996; 329:288-299.

9 de Alava E, Pardo J. Ewing tumor: tumor biology and clinical applications. Int J Surg Pathol 2001;9:7-17.

10 de Alava E, Gerald WL. Molecular biology of the Ewing's sarcoma/primitive neuroectodermal tumor family. J Clin Oncol 2000;18:204-213.

11 Ladanyi M. The emerging molecular genetics of sarcoma translocations. Diagnostic Mol Pathol 1995;4: 162-173.

$12 \mathrm{Gu}$ M, Antonescu CR, Guiter G, et al. Cytokeratin immunoreactivity in Ewing's sarcoma: prevalence in 50 cases confirmed by molecular diagnostic studies. Am J Surg Pathol 2000;24:410-416.
13 Collini P, Sampietro G, Bertulli R, et al. Cytokeratin immunoreactivity in 41 cases of ES/PNET confirmed by molecular diagnostic studies. Am J Surg Pathol 2001;25:273-274.

14 Srivastava A, Rosenberg AE, Selig M, et al. KeratinPositive Ewing's Sarcoma: An Ultrastructural Study of 12 Cases. Int J Surg Pathol 2005;13:43-50.

15 Moll R, Lee I, Gould VE, Berndt R, Roessner A, Franke WW. Immunocytochemical analysis of Ewing's tumors. Patterns of expression of intermediate filaments and desmosomal proteins indicate cell type heterogeneity and pluripotential differentiation. Am J Pathol 1987; 127:288-304.

16 Takayama S, Sugawa I. Electron microscopic observation of Ewing's sarcoma-a case report. Acta Pathol Japonica 1970;20:87-101.

17 Nusrat A, Parkos CA, Verkade P, et al. Tight junctions are membrane microdomains. J Cell Sci 2000;113: 1771-1781.

18 Heiskala M, Peterson PA, Yang Y. The roles of claudin superfamily proteins in paracellular transport. Traffic 2001;2:93-98.

19 Cereijido M, Anderson J. Tight Junctions, 2nd edn. CRC Press: Boca Raton, 2001.

20 Saitou M, Furuse M, Sasaki H, et al. Complex phenotype of mice lacking occludin, a component of tight junction strands. Mol Biol Cell 2000;11: 4131-4142.

21 Furuse M, Hirase T, Itoh M, et al. Occludin: a novel integral membrane protein localizing at tight junctions. J Cell Biol 1993;123:1777-1788.

22 Ando-Akatsuka Y, Saitou M, Hirase T, et al. Interspecies diversity of the occludin sequence: cDNA cloning of human, mouse, dog, and rat-kangaroo homologues. J Cell Biol 1996;133:43-47.

23 Furuse M, Itoh M, Hirase T, et al. Direct association of occludin with ZO-1 and its possible involvement in the localization of occludin at tight junctions. J Cell Biol 1994;127:1617-1626.

24 Fanning AS, Jameson BJ, Jesaitis LA, et al. The tight junction protein ZO-1 establishes a link between the transmembrane protein occludin and the actin cytoskeleton. J Biol Chem 1998;273:29745-29753.

25 Saitou M, Ando-Akatsuka Y, Itoh M, et al. Mammalian occludin in epithelial cells: its expression and subcellular distribution. Eur J Cell Biol 1997;73: 222-231.

26 Itoh M, Nagafuchi A, Yonemura S, et al. The 220-kD protein colocalizing with cadherins in non-epithelial cells is identical to ZO-1, a tight junction-associated protein in epithelial cells: cDNA cloning and immunoelectron microscopy. J Cell Biol 1993;121: 491-502.

27 Howarth AG, Hughes MR, Stevenson BR. Detection of the tight junction-associated protein ZO-1 in astrocytes and other nonepithelial cell types. Am J Physiol 1992;262:C461-C469.

28 Howarth AG, Singer KL, Stevenson BR. Analysis of the distribution and phosphorylation state of ZO-1 in MDCK and nonepithelial cells. J Membr Biol 1994; 137:261-270.

29 Howarth AG, Stevenson BR. Molecular environment of ZO-1 in epithelial and non-epithelial cells. Cell Motil Cytoskeleton 1995;31:323-332.

30 Billings SD, Walsh SV, Fisher C, et al. Aberrant expression of tight junction-related proteins ZO-1, claudin-1 and occludin in synovial sarcoma: an 
immunohistochemical study with ultrastructural correlation. Mod Pathol 2004;17:141-149.

31 Huber O. Structure and function of desmosomal proteins and their role in development and disease. Cell Mol Life Sci 2003;60:1872-1890.

32 Moll R, Cowin P, Kapprell HP, et al. Desmosomal proteins: new markers for identification and classification of tumors. Lab Invest 1986;54:4-25.

33 Garrod DR, Merritt AJ, Nie Z. Desmosomal adhesion: structural basis, molecular mechanism and regulation (Review). Mol Membr Biol 2002;19:81-94.
34 Perez-Moreno M, Jamora C, Fuchs E. Sticky business: orchestrating cellular signals at adherens junctions. Cell 2003;112:535-548.

35 Blaschuk OW, Rowlands TM. Plasma membrane components of adherens junctions (Review). Mol Membr Biol 2002;19:75-80.

36 Sanceau J, Truchet S, Bauvois B. Matrix metalloproteinase- 9 silencing by RNA interference triggers the migratory-adhesive switch in Ewing's sarcoma cells. J Biol Chem 2003;278:36537-36546. 